\title{
Comparison between Lacunary and Saturated Keggin POMs as Steel Corrosion Inhibitors in Chloride Solution: Contribution of the Lacuna in the Inhibition Process and Elucidation of the Protection Mechanism
}

\author{
Odilon Romaric Tchio Wamba, ${ }^{[a]}$ Martin Pengou, ${ }^{[b]}$ Cédric Baumier, ${ }^{[c]}$ Sylvain Franger, ${ }^{[d]}$ \\ Anne-Lucie Teillout, ${ }^{[\mathrm{e}]}$ Israël Martyr Mbomekallé, ${ }^{[\mathrm{e}]}$ Pedro De Oliveira, ${ }^{[\mathrm{e}]}$ Charles Péguy \\ Nanseu Njiki *[a] and Emmanuel Ngameni [a]
}

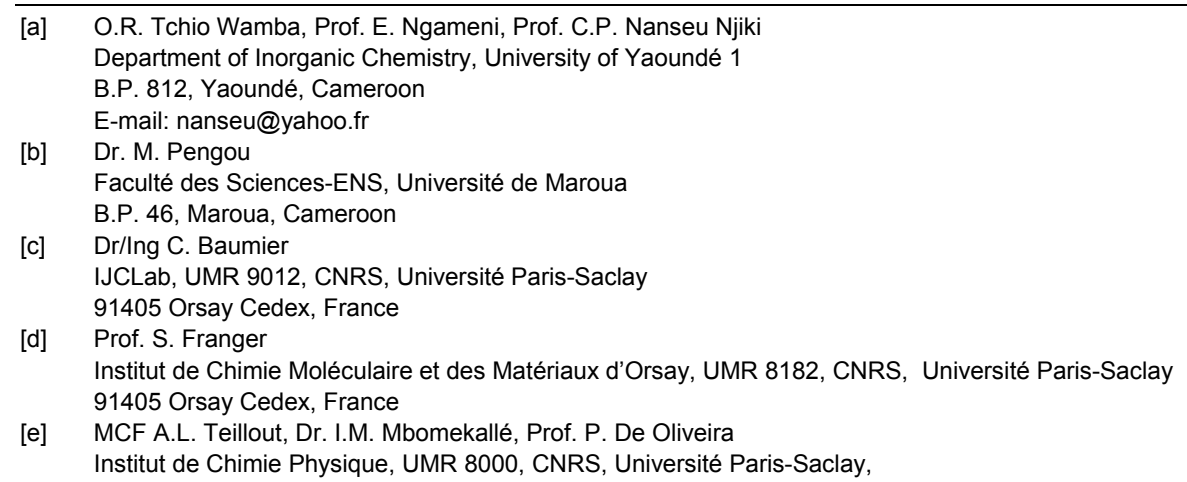

$\left(\mathrm{K}_{7}\left[\mathrm{PW}_{11} \mathrm{O}_{39}\right] \cdot 14 \mathrm{H}_{2} \mathrm{O}\right.$ and $\left.\mathrm{K}_{9}\left[\mathrm{AlW}_{11} \mathrm{O}_{39}\right] .15 \mathrm{H}_{2} \mathrm{O}\right)$ on the corrosion inhibition of $\mathrm{XC} 38$ steel in $\mathrm{NaCl} 0.1 \mathrm{M}$ was investigated. Electrochemical methods (polarization curves and electrochemical impedance spectroscopy) showed that the presence of controlled amounts of these compounds improved protection of the steel against corrosion. These results were supported by surface analysis, where only a minor amount of rust was produced on metal surface in the presence of polyoxometalates (POMs). The lacunary POMs were more efficient compared to their saturated species, highlighting the key role played by the lacuna on the inhibition process. Efficiency of POMs (both saturated and lacunary) was explained by their ability to increase the Fe(II)/Fe(III) molar ratio in greenish protective oxide layer formed upon steel oxidation. The mechanism proposed clearly explains the higher efficiency observed with lacunary POMs, in relation to their ability to react with free Fe(III) to yield a substituted structure.

\section{Introduction}

Iron as carbon steel is the most extensively used metal despite its poor resistance to oxidative degradation when exposed to the ambient environment. This degradation is mainly due to the action of oxygen as corrosion agent. Its action results in the metal surface covered by films of oxides/hydroxides that reduce somehow the corrosion rate, acting as a barrier that prevents the access of oxygen to the metal surface. The structures and the efficiency of these films in corrosion inhibition are strongly governed by the nature of the anions present in the corrosive medium. ${ }^{[1]}$ In the case of chlorides, these layers offer minor protection as a result of the localised solubilisation of the protecting layer following the complexation of iron cations by free chloride anions. This causes insidious and highly devastating pitting corrosion. Several approaches have been applied to minimise this form of corrosion. To date, the most efficient solution is certainly the use of corrosion inhibitors that can stabilise and reinforce the oxide/hydroxide layers on the steel surface. ${ }^{[1-4]}$ Oxoanion compounds of the $M O_{n}^{x-}$ type are commonly employed for this purpose. They act by adsorption to repair weak points or pores in the oxide film and they have oxidant properties in some cases. ${ }^{[1,3,5-7]}$ Most of these compounds, such as chromate and nitrite, are increasingly prohibited due to their acute toxicity. ${ }^{[8]}$ To address this issue, several works have been recently focused on the use of polyoxometalates (POMs) as an eco-friendly alternative of oxoanions as corrosion inhibitors. ${ }^{[9-12]}$

POMs are metal-oxo cluster anions based on early transition metals (e.g. Mo, W, V) in a high oxidation state. These complexes are formed in acid medium by self-assembly of polyhedral oxometallic units whose centres are occupied by a metallic atom $(\mathrm{M})$ and the vertices by oxygen atoms. The most common units are octahedra $\left(\mathrm{MO}_{6}\right)$ and tetrahedra $\left(\mathrm{MO}_{4}\right)$. Some POMs are synthesized in neutral or alkaline solutions depending on the desired final configuration of the targeted compound. ${ }^{[13]}$ POMs are known to accept electrons without major changes of their structures and they form insoluble salts with large cations. They found many applications in various fields such as health care, catalysis and environmental protection. ${ }^{[14-16]}$ Several works also showed that, in the line of their physicochemical properties, they are particularly attractive as oxidizing and film-forming corrosion inhibitors in concentrated chloride aqueous solutions. ${ }^{[7]}$ In 1994 , 
Lomakina et al. showed that heteropolytungstates can effectively prevent aluminium corrosion by reducing the rate of aluminium oxide film formation. ${ }^{[17]}$ Bonastre et al. reported the use of an electro-synthesised polypyrrole / $\left[\mathrm{PW}_{12} \mathrm{O}_{40}\right]^{3-}$ hybrid material for the protection of carbon steel electrodes against corrosion. ${ }^{[18]}$ In addition to the above saturated Keggin or Dawson-type POMs, substituted POMs and POM-based inorganic-organic hybrid materials, also showed good corrosion inhibition performances. ${ }^{[9-11,19,20]}$ It is generally accepted that the inhibition performance of these POMs is based on their oxidising power and on the nucleophilic nature of the terminal oxygens of their structures. The oxidising power is expected to promote the fast formation of a more stable oxide layer ${ }^{[17]}$ readily stabilised and reinforced by the adsorption of the nucleophilic terminal oxygen atoms of POMs. ${ }^{[3]}$

Despite these results, almost no work was dedicated to the elucidation of the mechanism that governs the action of POMs during the inhibition of corrosion of steel in chloride media. This particularly includes the interactions of POMs with dissolved oxygen, which actually constitutes the real direct corrosion agent. The role of the interactions between the nucleophilic oxygen atoms and the protective layer also remains to be clarified. An interesting solution may be the comparison of the inhibitory efficiency of POMs, which differs only in the nucleophilic nature of the terminal oxygen atoms. Lacunary POMs are a class of compounds characterised by one or more missing tungstate subunits. The oxygen atoms surrounding the lacuna therefore present a more marked nucleophilic character. This explains the reported high reactivity of lacunary POMs with respect to certain metals $(\mathrm{Ag}, \mathrm{Au}, \mathrm{Pt})$ and with some metallic cations $\left(\mathrm{Fe}^{3+}, \mathrm{Cu}^{2+}, \mathrm{Mn}^{2+}\right) \cdot{ }^{[21,22]}$

The objective of this work is to compare the efficiency of saturated POMs and their unsaturated counterparts in order to elucidate the mechanism governing the action of these compounds as steel corrosion inhibitors in chloride aqueous solution. Practically, comparative studies were performed between two lacunary POMs $\left(\mathrm{K}_{7}\left[\mathrm{PW}_{11} \mathrm{O}_{39}\right] .14 \mathrm{H}_{2} \mathrm{O}\right.$ and $\mathrm{K}_{9}\left[\mathrm{AIW}_{11} \mathrm{O}_{39}\right] .15 \mathrm{H}_{2} \mathrm{O}$ respectively named PW11 and AIW11) and their saturated equivalents $\left(\mathrm{H}_{3}\left[\mathrm{PW}_{12} \mathrm{O}_{40}\right] .14 \mathrm{H}_{2} \mathrm{O}\right.$ and $\mathrm{H}_{5}\left[\mathrm{AIW}_{12} \mathrm{O}_{40}\right] \cdot 15 \mathrm{H}_{2} \mathrm{O}$ respectively named PW12 and AIW12). Several electrochemical methods (Open Circuit Potential, Electrochemical Impedance Spectroscopy (EIS), linear polarisation) were used to collect useful information about the corrosion rate, together with surface characterisation for visual appreciation of steel corrosion.

\section{Results and Discussion}

Prior to the electrochemical experiments, some investigations were performed in order to check the stability of the POMs in the experimental conditions of the corrosion tests. Cyclic voltammograms and UV-visible spectra of POM samples were recorded as a function of time in $\mathrm{NaCl} 0.1 \mathrm{M}$. The results obtained confirmed the stability of the metal oxides in the electrolytic solution (see supplementary information, Figures S1 and S2).

\section{Chronopotentiometry: Open Circuit Potential (OCP)}

Open circuit potential monitoring provides preliminary information on the nature of the processes occurring at the metal/electrolyte interface (corrosion, passivation...) and on the required immersion time for the establishment of a stationary regime. The achievement of this equilibrium is mandatory before performing linear polarisation and electrochemical impedance measurements. ${ }^{[23]}$ The curves obtained are presented in Figure 1.

In $0.1 \mathrm{M} \mathrm{NaCl}$ solution (blank solution), the OCP of the XC38 steel decreases sharply during the first $2000 \mathrm{~s}$ due to the active corrosion of the metal. This was followed by a slight and constant decrease of the potential due to the gradual stabilisation of the corrosion rate. After two hours, the recorded OCP was $-587 \mathrm{mV}$.

When the electrolyte contains a controlled amount of lacunary PW11, a sharp decrease of potential during the first 500 seconds was recorded, followed by a slight and constant decrease. This indicates that the presence of lacunary POMs promotes a fast stabilisation of the potential at the steel surface. In addition, the chronopotentiometry curve displayed potentials systematically higher than those recorded without the POM in solution, a proof that even minor amounts of this compound were able to provide valuable protection against steel corrosion. Only minor changes were observed when the POM concentration was varied up to $100 \mathrm{ppm}$ (the OCP values at the end of the measurements were in the range -502 $\mathrm{mV}$ to $-503 \mathrm{mV}$ in the presence of PW11 at concentrations of 25, 50 and $100 \mathrm{ppm}$ ). A similar trend was obtained with the corresponding saturated POM (PW12), but with a smaller efficiency as confirmed by the more negative anodic values recorded for the OCP. This similar trend can be attribute to fact that, in this experimental condition, PW12 partially transform in PW11. It is known that PW12 is quite instable in pH range more than 3 and the $\mathrm{pH}$ of the solution containing 100 ppm of PW12 was 3.67. At this pH around $20 \%$ of PW12 is transformed in PW11 in 24 h. ${ }^{[24]}$

In the presence of AIW11, the OCP values followed similar trends as those recorded when PW11 was used in the corrosive solution. However, it can be noted that this compound seems less effective because at a concentration of $25 \mathrm{ppm}$, the shape of the curve was intermediate between that obtained in the absence of $\mathrm{POM}_{S}$ and those recorded in the presence of $50 \mathrm{ppm}$ of AIW11. The saturated analogue of this POM (AIW12) showed a poor effect on the OCP, since the curve obtained was close to that of the control experiment recorded without the POM. 

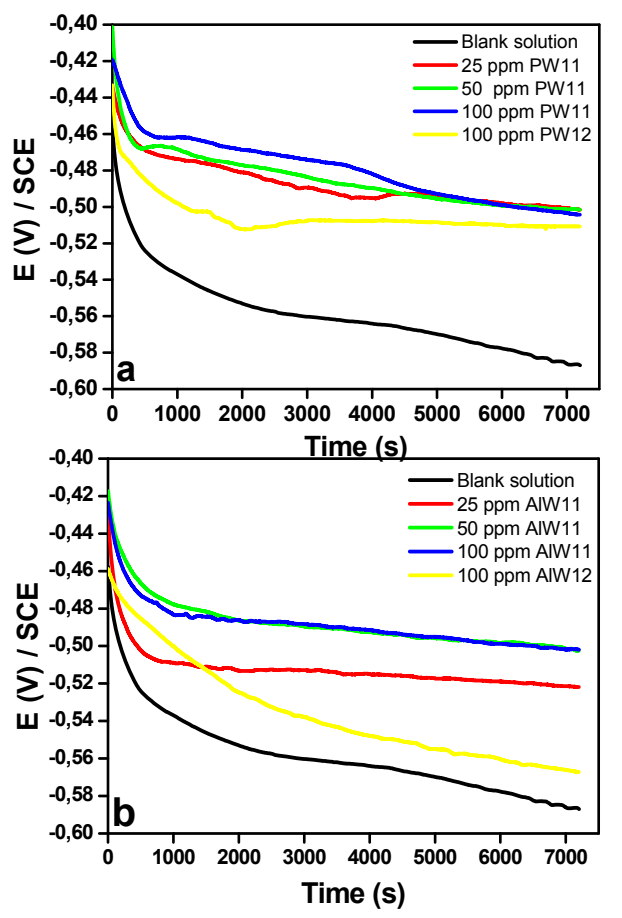

Figure 1. Chronopotentiometry curves of XC38 mild steel in $0.1 \mathrm{M} \mathrm{NaCl}$ solution in the presence and in the absence of lacunary POMs (PW11 and AIW11) and of saturated POMs (PW12 and AIW12)

\section{Polarisation curves}

The polarisation curves of steel XC38 in $0.1 \mathrm{M} \mathrm{NaCl}$ containing lacunary POMs and saturated POMs at room temperature $\left(20^{\circ} \mathrm{C}\right)$ are presented in Figure 2 . The data about corrosion current and corrosion potential obtained from the extrapolation of Tafel lines are summarised in Table 1.

The presence of either PW11 (Figure 2a) or AIW11 (Figure 2b) for all the tested concentrations results in a decrease of the anodic current densities and a shift of the corrosion potential towards anodic values (more than $75 \mathrm{mV}$ ). This behaviour further confirms the anodic inhibitor character of the lacunary POMs tested. ${ }^{[3,25]}$ For potentials above $-0.3 \mathrm{~V}$, a fast and significant increase in the oxidation current was observed in the presence of lacunary POMs. This behaviour is generally attributed to the destruction or the desorption of a protective layer anchored on the metal surface. ${ }^{[26]}$ On the other hand, a slight increase in the cathodic current was observed in the presence of inhibitors and attributed to the direct electrochemical reduction of POMs on the metal surface. Such behaviour was frequently reported when compounds with oxidising properties were used as corrosion inhibitors in a medium close to $\mathrm{pH}$ neutrality. ${ }^{[3]}$ To confirm the ability of POMs to reduce on the steel surface in $\mathrm{NaCl}$ solution, some experiments were carry out in deaerated solution of $\mathrm{NaCl} 0.1 \mathrm{M}$. The presence of POMs in solution resulted in a considerable increase of the cathodic current with the concentration of POMs, thus reflecting the fact that the POM's reduction control the cathodic current. A slight increase in the anodic current was also observed, proving that the reduction of POM leads to steel oxidation. These results are presented in Figure S3 (see supplementary information). This behaviour of $\mathrm{POMs}$ in deaerated $\mathrm{NaCl}$ medium makes it possible to say that the inhibition of steel XC38 in $\mathrm{NaCl}$ medium in the presence of POMs requires the presence of oxygen. Oxygen would therefore intervene in the inhibition mechanism.

The presence of saturated POMs (PW12 and AIW12) also results in protection against corrosion, but to a lesser extent compared to their lacunary counterparts at equal concentration (Figure 2). The shift of corrosion potential to more positive value is also observed in the case of saturated POMs. This similarity with lacunary POMs would allow to say that the inhibition mechanisms of these POMs would be almost identical even if the action of lacunary POMs seem much more marked. Inhibition percentage values of $56.4 \%$ and $39.6 \%$ for 100 ppm of PW11 and PW12 respectively, and of $61.8 \%$ and $33.3 \%$ for $100 \mathrm{ppm}$ of AIW11 and AIW12 respectively, were obtained from the experimental values (Table 1). Inhibition percentage values obtained from the polarisation resistance $\left(n_{R p}\right)$ follow similar trends with minor variations compared to $\eta_{\text {Icorr }}$ values. These results confirm that the presence of the lacuna promotes corrosion inhibition. A close observation of the polarisation curves recorded in the presence of saturated POMs also showed that the protective film desorption (above $-0.3 \mathrm{~V}$ ) was poorly defined. ${ }^{[27]}$ 


\section{Electrochemical Impedance Spectroscopy}

Figures 3a and 3b present the Nyquist diagrams of XC38 steel in $0.1 \mathrm{M} \mathrm{NaCl}$ in the presence of controlled amounts of POMs. The presence of POMs in solution increases the diameter of the capacitive loops, indicating an effective protection against corrosion provided by these compounds. The precise estimation
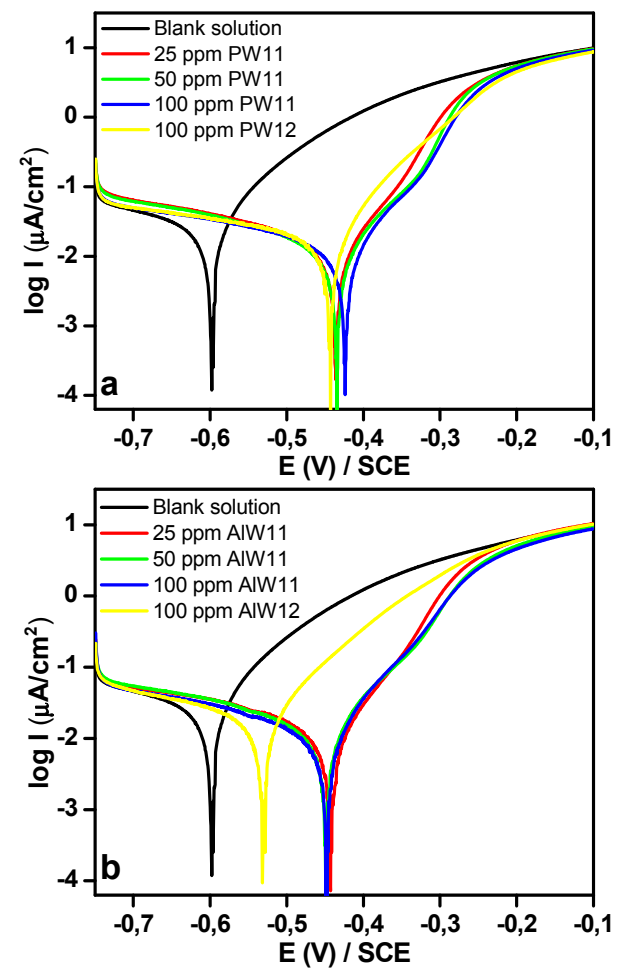

Figure 2. Polarisation curves of $X C 38$ mild steel in $0.1 \mathrm{M} \mathrm{NaCl}$ in the absence and in the presence of lacunary POMs (PW11 and AIW11) and of saturated POMs (PW12 and AIW12)

Table 1. Kinetic parameters obtained from polarisation curves of XC38 mild steel in $0.1 \mathrm{M} \mathrm{NaCl}$ in the absence and in the presence of different concentrations of PW11, AlW11, PW12 and AIW12.

$\left(\Delta E=\right.$ Ecorr $_{\text {Inhibitor }}-$ Ecorr $\left._{\text {Blank solution }}\right)$

\begin{tabular}{|c|c|c|c|c|c|c|c|}
\hline Inhibitor & $\begin{array}{l}\text { Amount } \\
\text { (ppm) }\end{array}$ & $\begin{array}{l}\text { Ecorr } \\
\text { (mV/ } \\
\text { Ref) }\end{array}$ & $\begin{array}{l}\Delta \mathrm{E} \\
\text { (mV/Ref) }\end{array}$ & $\begin{array}{l}\text { Icorr } \\
\left(\mu \mathrm{A} / \mathrm{cm}^{2}\right)\end{array}$ & $\begin{array}{l}\mathrm{Rp} \\
\left(\Omega . \mathrm{cm}^{2}\right)\end{array}$ & $\begin{array}{l}\eta_{\text {Icorr }} \\
(\%)\end{array}$ & $\begin{array}{l}\eta_{\mathrm{Rp}} \\
(\%)\end{array}$ \\
\hline \multirow{4}{*}{ PW11 } & 0 & -600 & 0 & 29.8 & 939 & - & - \\
\hline & 25 & -438 & 162 & 13.6 & 1979 & 54.4 & 52.6 \\
\hline & 50 & -436 & 164 & 11.6 & 2284 & 61.1 & 58.9 \\
\hline & 100 & -426 & 174 & 13.0 & 2107 & 56.4 & 55.4 \\
\hline \multirow{4}{*}{ AlW11 } & 0 & -600 & 0 & 29.8 & 939 & - & - \\
\hline & 25 & -453 & 147 & 13.9 & 1979 & 53.4 & 52.6 \\
\hline & 50 & -452 & 148 & 13.3 & 2104 & 55.4 & 55.4 \\
\hline & 100 & -450 & 150 & 11.4 & 2411 & 61.8 & 61.1 \\
\hline \multirow{3}{*}{ PW12 } & 0 & -600 & 0 & 29.8 & 939 & - & - \\
\hline & 50 & -449 & 151 & 15.9 & 1787 & 46.6 & 47.5 \\
\hline & 100 & -445 & 155 & 18.0 & 1421 & 39.6 & 33.9 \\
\hline \multirow{3}{*}{ AIW12 } & 0 & -600 & 0 & 29.8 & 939 & - & - \\
\hline & 50 & -505 & 95 & 19.2 & 1506 & 35.6 & 37.6 \\
\hline & 100 & -533 & 67 & 19.8 & 1406 & 33.3 & 33.2 \\
\hline
\end{tabular}


of this corrosion protection ability was based on electrochemical parameters such as the charge transfer resistance (Rct), the resistance of the surface film (Rf), the solution resistance $(\mathrm{Re})$ and the constant phase elements (CPE) parameters: $\mathrm{Y}_{0}$ (admittance constant) associated to the capacity of the double layer (Cdl), and $\alpha$ (surface homogeneity parameter). These parameters were equivalent electrical circuits (EEC). The EEC use in this study was inserted in the Nyquist diagram. This circuit was proposed assuming that two time constants (the electrical double layer and the iron oxide layer) would govern the processes at the interface, which is consistent with the presence of two inflection points in the corresponding Bode diagrams, according to a Phase Inflection Detection (PID) method as described in ${ }^{28]}$. These electrical parameters for different experimental conditions are compiled in Table 2. In order to derive real capacitance values from the CPE corresponding to the double layer capacitance used in the impedance model, the Brug's relationship was used (Equation 1) ${ }^{[29,30]:}$

$C_{d l}=Y_{0}^{\frac{1}{\alpha}}\left(R_{e}^{-1}+R_{c t}{ }^{-1}\right)^{\frac{\alpha-1}{\alpha}}$

The parameters used in equation 1 are defined above.

With regard to Table 2, the Cdl values obtained in the presence of lacunar POMs are lower than those obtained in blank solution. This behaviour further confirm a reduction of local electricity at the interface in the presence of lacunar POMs and consequently an increase of the charge transfer resistance ${ }^{[31]}$. On the other hand, an increase in Cdl is recorded in presence of saturated POM, despite the slight increase of Rct (low percentage of inhibition). A similar observation was made by made by Kakaei and others in 2019 [30]. This evolution of Cdl in the presence of saturated POMs can be firstly attributed to the electroactive nature of the POM. At the potential where EIS experiment is carried out, POM can be reduced electrochemically thus causing an intensification of the charge transfer at the interface. It is also possible to attribute this increase of $\mathrm{Cdl}$ to the porous and not protective character of the surface film formed in the presence of saturated POMs. Like their saturated equivalent, lacunar POMs are also electroactive, but they have the possibility to form surface compounds with metal cations $\left(\mathrm{Fe}^{2+}\right.$ and $\mathrm{Fe}^{3+}$ ) due to their higher anionic charge and the strong nucleophilic character of the oxygen atoms present at the level of lacuna. This specificity of the lacunar POMs can then be correlated to the better inhibition percentages obtained in Table 2 (64.8\% and $67.1 \%$ for 100 ppm of PW11 and 100 ppm of AIW11 respectively).

The Bode diagrams for XC38 steel in the absence and in the presence of POMs are presented in Figures $3 c, 3 d, 3 e$ and $3 \mathrm{f}$. In the presence of POMs in the corrosive solution, two inflection points clearly appear on the phase diagrams, even at low concentration, in agreement with the existence of two time constants included in the equivalent circuit, and also confirming the formation of a stable film on the steel surface in the presence of POMs. On the Bode phase diagram recorded without inhibitor, the curve exhibits only one inflection point, indicating the presence of a unique time constant. The latter would be mainly related to the electrical double layer. It is thus possible to conclude that the oxide layer formed on the steel surface in $0.1 \mathrm{M} \mathrm{NaCl}$ medium in the absence of POMs is either rough or very porous (as stated by the low $\alpha$ coefficient standing for an inhomogeneous exposed surface), but in all cases poorly protective and not really influencing the kinetics of the electron transfer on the metal/solution interface. This result is also in agreement with the facile and fast solubilisation of the oxide layer by chloride anions. In fact, the oxide layer that forms in the absence of inhibitor has a low $\mathrm{Fe}(\mathrm{II}) / \mathrm{Fe}(\mathrm{III})$ ratio, which would then make it easier for the layer to be attacked by chloride ions. 

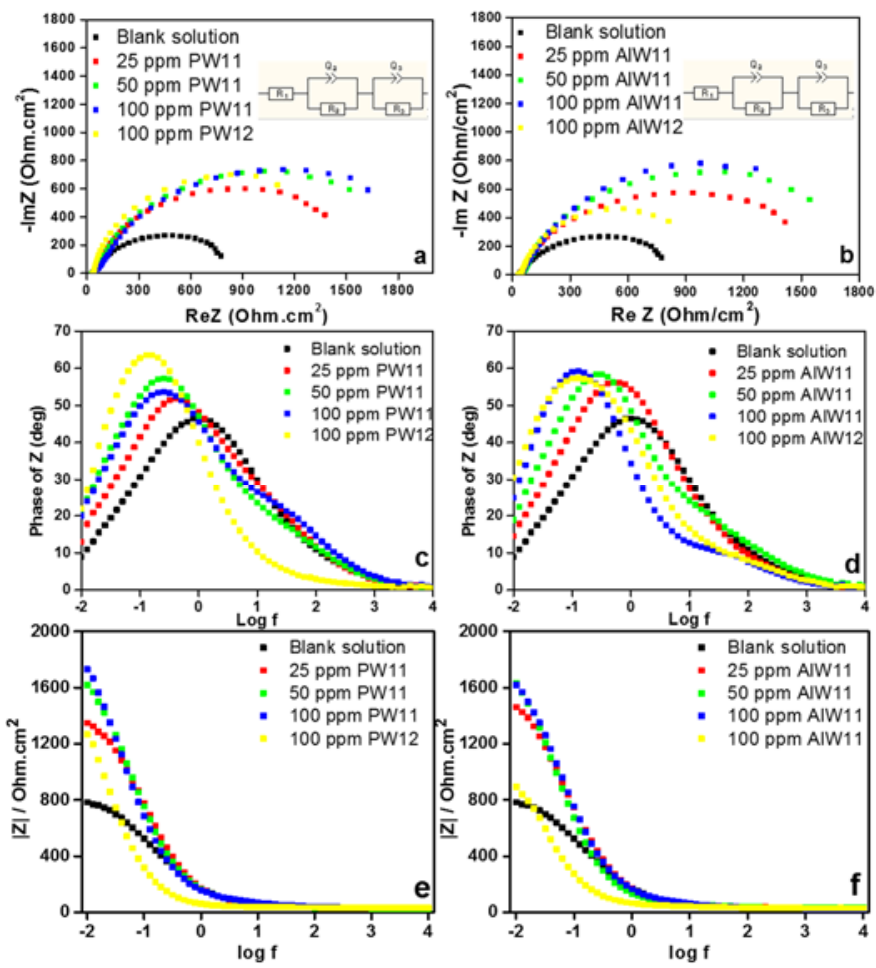

Figure 3. Nyquist ( $a$ and b) and Bode diagrams (c, d, e and f) of XC38 mild steel in the absence and in the presence of lacunary and saturated POMs

Table 2. Electrochemical parameters obtained from the Nyquist diagrams for $\mathrm{XC} 38$ mild steel in $0.1 \mathrm{M} \mathrm{NaCl}$ in the absence and in the presence of PW11, AIW11, PW12 and AIW12

\begin{tabular}{|c|c|c|c|c|c|c|}
\hline Inhibitor & $\begin{array}{c}\text { Amount } \\
\text { (ppm) }\end{array}$ & $\begin{array}{c}\mathbf{R}_{\mathrm{ct}} \\
\left(\Omega . \mathrm{cm}^{2}\right)\end{array}$ & $\alpha_{d c}$ & $\begin{array}{c}\mathrm{C}_{\mathrm{dl}} \\
\left(\mathrm{mF} \cdot \mathrm{cm}^{-2}\right)\end{array}$ & $\begin{array}{c}R_{\mathrm{f}} \\
\left(\Omega . \mathrm{cm}^{2}\right)\end{array}$ & $\eta(\%)$ \\
\hline \multirow{4}{*}{ PW11 } & 0 & 729 & 0,66 & 1.63 & - & - \\
\hline & 25 & 1438 & 0.82 & 0.41 & 25.7 & 49.3 \\
\hline & 50 & 1915 & 0.82 & 0.77 & 28.1 & 62.0 \\
\hline & 100 & 2072 & 0.81 & 0.37 & 38.8 & 64.8 \\
\hline \multirow{4}{*}{ AIW11 } & 0 & 729 & 0,66 & 1.63 & - & - \\
\hline & 25 & 1622 & 0.79 & 0.68 & 8.6 & 55.0 \\
\hline & 50 & 1844 & 0.84 & 0.75 & 33.4 & 60.5 \\
\hline & 100 & 2216 & 0.79 & 0.43 & 14.6 & 67.1 \\
\hline \multirow{3}{*}{ PW12 } & 0 & 729 & 0,66 & 1.63 & - & - \\
\hline & 50 & 1486 & 0.84 & 1.97 & 24.5 & 50.9 \\
\hline & 100 & 1616 & 0.90 & 3.96 & 9.7 & 54.9 \\
\hline \multirow{3}{*}{ AIW12 } & 0 & 729 & 0,66 & 1.63 & - & - \\
\hline & 50 & 1124 & 0.86 & 3.66 & 8.5 & 35.1 \\
\hline & 100 & 1104 & 0.88 & 4.43 & 17.5 & 34.0 \\
\hline
\end{tabular}

\section{Surface analyses}

Images of the steel surface after $5 \mathrm{~h}$ of immersion in $0.1 \mathrm{M} \mathrm{NaCl}$ without and with the addition of $100 \mathrm{ppm}$ of PW11, 100 ppm of AIW11, 100 ppm of PW12 and 100 ppm of AIW12 in the electrolyte are presented in Figure 4. Without POM (Figure 4a) the electrode surface was covered by large brownish and dark areas, resulting from iron oxidation. This clearly confirmed that steel undergoes almost a general corrosion in $0.1 \mathrm{M} \mathrm{NaCl}$ aqueous solution in the absence of inhibitors. When PW11 or AIW11 were added to the corrosive solution, greenish spots and areas were observed on the metal surface. In the presence of PW12, the layer formed on the steel surface is a very dark green, proof that it would be less protective than the film formed in the presence of PW11. Indeed, the partial transformation of PW12 into PW11 under these experimental conditions may justify the appearance of this green coloration, which can be attributed to the action of the small amount of PW11 formed. In the presence of AlW12, the dark areas reappear even if they are not distributed in the same way as in the absence of inhibitors. This difference in behaviour may explain the inhibitory efficiency observed with lacunary POMs. 
The SEM images and the EDX profiles of XC38 steel samples in $0.1 \mathrm{M} \mathrm{NaCl}$ solution after $5 \mathrm{~h}$ of immersion in the absence and in the presence of POMs are presented in Figure 5. Two POMs were selected for surface characterisation, PW11 (lacunary POM with the highest inhibitory efficiency) and AIW12 (saturated POM with the lowest inhibitory efficiency).

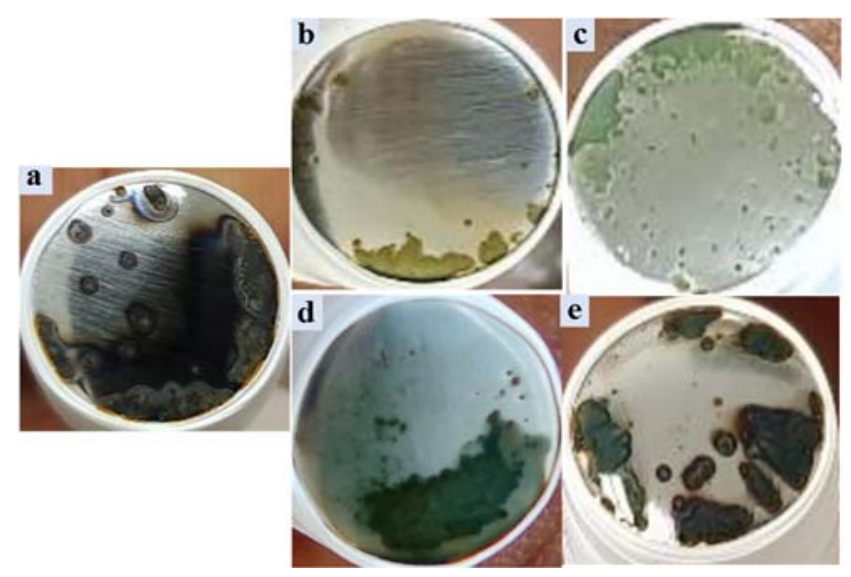

Figure 4. Images of the surface of the steel XC38 in $\mathrm{NaCl}$ medium (0.1M) without inhibitor (a), and in the presence of 100 ppm of PW11 (b), 100 ppm of AlW11 (c), 100 ppm PW12 (d) and 100 ppm of AIW12 (e)

In Figure $5 a$ (Steel XC38 in $0.1 \mathrm{M} \mathrm{NaCl}$ without POM), severe damage is visible on the metal surface with many cracks and pits caused by chloride ions. ${ }^{[1,3,27,32]}$ It is known that aggressive anions such as chloride cause the breakdown of the passive oxide film at weak points on the metal surface. The corrosion rate is generally high at these weak points compared to the rest of the passive surface. EDX analysis of the steel surface showed an almost general corrosion with the concomitant formation of an oxide layer according to the proportions of iron and oxygen on the scanned surface. However, this oxide layer must be porous because of pitting and cracking and therefore poorly protective against corrosion.

On the other hand, a scan of the steel surface in the presence of PW11 and of AIW12 has two main zones (1 and 2 in Figures $5 b$ and $5 c$ ). Zone 1 is characteristic of an added layer and corresponds to the visible deposit on the steel contours in the presence of POM. Zone 2 shows a surface almost intact and free from all forms of corrosion. Very few localised corrosion sites are visible on the steel surface in the presence of POM, which probably inhibits pitting corrosion. The pitting inhibition action of the POM may be achieve by: (i) improving the protective nature of the film by changing its composition and structure; (ii) reducing the imperfections in the film; and (iii) reducing the extent of anion adsorption on the film by competitive adsorption of other chemical species. ${ }^{[3,33]}$ According to the high negative charge of POMs (which leads to a competitive adsorption between oxoanions and chloride anions) and their strong oxidizing abilities (which favour the formation of a more protective oxide layer), the three mechanisms are feasible. EDX analysis of zone 1 showed a fairly similar profile between iron and oxygen in the presence of POM, proof of the presence of an oxide layer. Iron is predominant in zone 2 of the steel surface, confirming that the latter remained almost intact. In the presence of the lacunary POM, the iron proportion on the steel surface is not very different from one zone to another. This would reflect less severe corrosion on the steel surface in this case. In contrast, when a saturated POM is used as inhibitor, an important decrease of the iron proportion was observed from zone 2 to zone 1. This result is in agreement with the fact that, in the presence of lacunary POMs, the oxide layers formed are more protective.
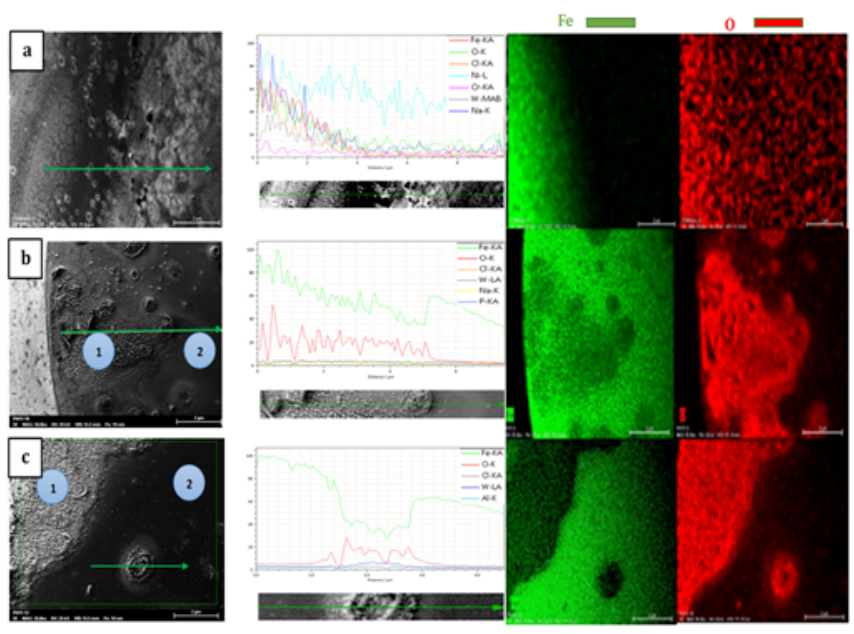
Figure 5. SEM, EDX profile and EDX mapping of the XC38 steel surface in $0.1 \mathrm{M} \mathrm{NaCl}$ medium without inhibitor (a), and in the presence of 100 ppm PW11 (b) and 100 ppm AIW12 (c)

\section{Mechanism of protection against corrosion by POMs Elucidation of the difference in behaviour between lacunary POMs and saturated POMs}

A mechanism governing the action of POMs as steel corrosion inhibitors was proposed based on experimental results previously described. Data obtained from the electrochemical studies and information on the reactivity of the POMs made it possible to propose a mechanism of action of these metal oxide complexes at the interface when they are used as corrosion inhibitors. To facilitate the presentation of the mechanism, it is appropriate to recall the mechanism of steel corrosion in a $\mathrm{NaCl}$ medium.

When steel is immersed in $\mathrm{NaCl}$ solution without $\mathrm{POM}$, the reaction that control cathodic current is the reduction of dissolved oxygen (Eq.2). ${ }^{[3]}$ By a redox process, this reaction induce the oxydation of iron to yield $\mathrm{Fe}$ (II) as iron (II) hydroxide. Since this hydroxide is not stable in the presence of oxygen at near neutral $\mathrm{pH}$, it is further oxidised to $\mathrm{FeOOH}$ (Scheme 1 (left), Eq.3):

$\mathrm{O}_{2}+2 \mathrm{H}_{2} \mathrm{O}+4 e^{-} \leftrightarrow 4 \mathrm{OH}^{-}$

$4 \mathrm{Fe}+3 \mathrm{O}_{2}+2 \mathrm{H}_{2} \mathrm{O} \rightarrow 4 \mathrm{FeOOH}$

Eq. 3

Practically, the product of this oxidation is a complex mixture of $\mathrm{Fe}(\mathrm{III})$ and $\mathrm{Fe}(\mathrm{II})$ oxides and oxyhydroxides $(\alpha-\mathrm{FeOOH}, \beta-$ $\mathrm{FeOOH}, \mathrm{Fe}_{2} \mathrm{O}_{3}, \mathrm{Fe}_{3} \mathrm{O}_{4}$ etc.). ${ }^{[34]}$ It is a black-to-rust mixture found on oxidised steel samples as shown in Figure 5a. This layer acts as a barrier that reduces the rate of access of dissolved oxygen to the metal surface, providing protection against corrosion. On the other hand, when the amount of $\mathrm{Fe}$ (III) is predominant with respect to that of $\mathrm{Fe}$ (II) (low $\mathrm{Fe}$ (II)/Fe(III) ratio), the protective layer is easily destroyed by chloride ions. ${ }^{[5]}$ As a result, dissolved oxygen can directly access the metal surface.

In summary, a good steel corrosion inhibitor in chloride medium at near neutral $\mathrm{pH}$ is then expected to promote the formation of $\mathrm{Fe}(\mathrm{II})$ rich protective layers and to reduce the concentration of dissolve oxygen at the vicinity of the metal surface.

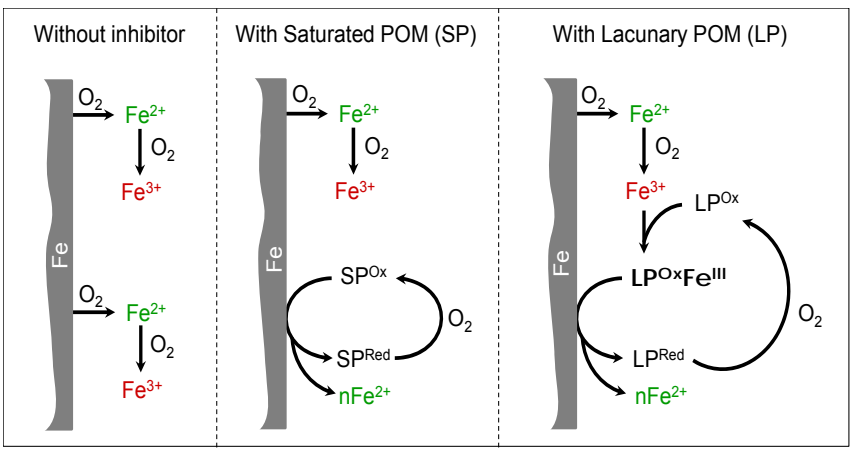

Scheme 1. Steel corrosion inhibition mechanism by POMs in $\mathrm{NaCl}$ medium

When the steel is immersed in $\mathrm{NaCl}$ solution in the presence of POMs, saturated or lacunary ones, two cathodic reactions take place on steel surface, the reduction of dissolved oxygen and the reduction of POM. Cathodic current is now control by these reactions. However, depending on whether the POM is saturated or lacunary, his reduction on the steel surface will have different consequences at the interface level.

In the presence of saturated POM $\left(\mathrm{SP}^{\mathrm{Ox}}\right)$ in the corrosive solution. The $\mathrm{SP}^{\mathrm{Ox}}$ oxidises iron to $\mathrm{Fe}(\mathrm{II})$ and its reduced form (SPRed) reacts locally with dissolved $\mathrm{O}_{2}$, $\mathrm{SP}^{\mathrm{Ox}}$ being regenerated as shown in Eq.4 and 5. ${ }^{[17,19,36]}$ The cycle can carry on without $\mathrm{O}_{2}$ directly accessing the electrode surface. In areas not accessible by the POM, $\mathrm{Fe}(\mathrm{II})$ is directly oxidised to $\mathrm{Fe}(\mathrm{III})$ by $\mathrm{O}_{2}$. Scheme 1 (middle) summarises this mechanism.

$S P^{O x}+n F e \rightarrow S P^{R e d}+n F e(I I)$

Eq. 4

$S P^{R e d}+n \mathrm{O}_{2}+p \mathrm{H}_{2} \mathrm{O} \rightarrow S P^{O x}+m \mathrm{HO}^{-}$

Eq. 5

In the presence of the lacunar POM (LPOX), two pathways are possible: (i) LPOx directly oxidises the metal surface to yield $\mathrm{Fe}(\mathrm{II})$ and reduced lacunar POM (LPRed) (Eq 6); this highly reactive species is oxidised to $\mathrm{LP}^{\mathrm{Ox}}$ by dissolved $\mathrm{O}_{2}(\mathrm{Eq} .7)$; 
(ii) $\mathrm{LP}^{\mathrm{Ox}}$ reacts with $\mathrm{Fe}(\mathrm{III})$ produced by iron corrosion in the presence of $\mathrm{O}_{2}$ to yield a $\mathrm{Fe}(\mathrm{III})$ saturated POM (LP $\left.{ }^{\mathrm{Ox}} \mathrm{Fe}^{\mathrm{III}}\right)$ (Eq 8). Indeed, metal cations such as $\mathrm{Fe}^{3+}$ and $\mathrm{Cu}^{2+}$ are able to fill the lacuna of unsaturated POMs. ${ }^{[37]} \mathrm{LP}^{\mathrm{Ox}} \mathrm{Fe}^{\mathrm{III}}$ subsequently oxidises the metal to generate Fe(II) and the reduced lacunar POM (LPRed), as showed in Eq.9. Indeed, it is well known that during the reduction of $\mathrm{Fe}(\mathrm{III})$-substituted POMs, $\mathrm{Fe}(\mathrm{II})$ is released from the reduced substituted POM scaffold. ${ }^{[38]}$ This mechanism is illustrated in a simplified manner in scheme 1 (right).

$$
\begin{aligned}
& L P^{O x}+n \mathrm{Fe} \rightarrow L P^{R e d}+n \mathrm{Fe}(I I) \\
& L P^{R e d}+n \mathrm{O}_{2}+p \mathrm{H}_{2} \mathrm{O} \rightarrow \mathrm{LP} P^{O x}+m \mathrm{HO}^{-} \\
& L P^{O x}+\mathrm{Fe}(I I I) \leftrightarrow L P^{O x} \mathrm{Fe}^{I I I} \\
& L P^{o x} \mathrm{Fe} e^{I I I}+n \mathrm{Fe} \rightarrow L P^{\text {Red }}+(n+1) \mathrm{Fe}(I I)
\end{aligned}
$$

Eq. 7

Eq. 8

The presence of a POM in the corrosive solution clearly reduces the concentration of dissolved oxygen and $\mathrm{Fe}(\mathrm{III})$ compounds at the vicinity of the steel surface. This phenomenon is much more marked in the presence of a lacunary $\mathrm{POM}$, because of its peculiar ability to react with $\mathrm{Fe}(\mathrm{III})$ (Eq. 8). This contributes to increase the $\mathrm{Fe}(\mathrm{II}) / \mathrm{Fe}(\mathrm{III})$ ratio of the protective layer. Indeed, this type of oxide layer is known to be the protective against corrosion. ${ }^{[3,39]}$ The presence of greenish spots on the steel surface after immersion in the corrosive solution containing the lacunary POM fully supports this hypothesis. The action of lacunary POM (as steel corrosion inhibitor) would therefore be very marked at the level of the anodic processes by the fact that it promotes the formation of a protective oxide layer. This property therefore confirms its anodic inhibitor character, as shown by the polarization curves (fig 2). ${ }^{3]}$

In order to confirm this mechanism, $\mathrm{Fe}$ (III)- and $\mathrm{Cu}(\mathrm{II})$-substituted POMs (PW11Fe and PW11Cu) were tested as corrosion inhibitors of XC38 steel in $0.1 \mathrm{M} \mathrm{NaCl}$ medium. The results obtained in Figure 6 show a similar behaviour to that observed in the presence of the corresponding lacunary POM (PW11).
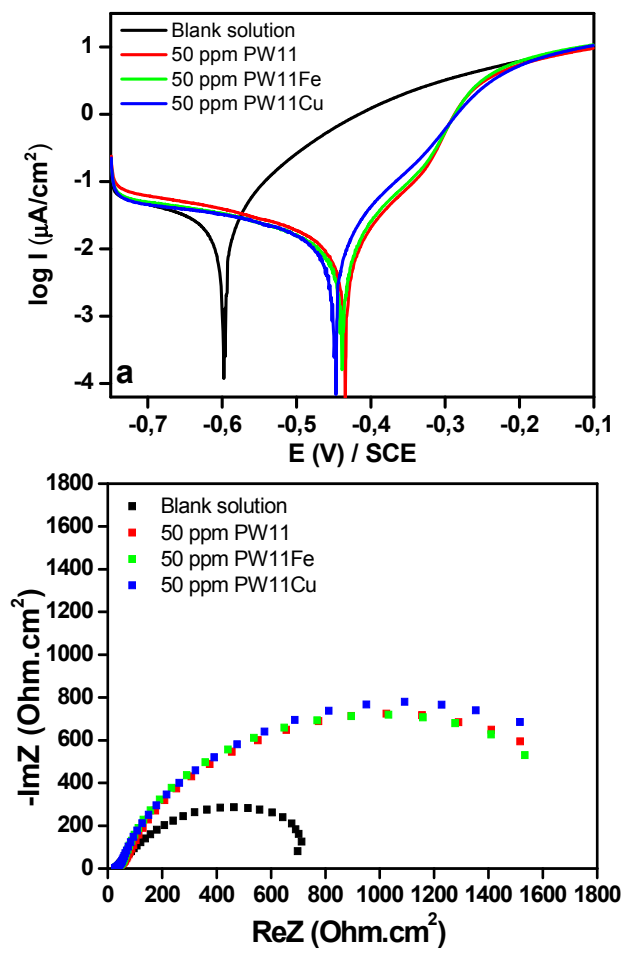

Figure 6. Polarisation curves and Nyquist plot of XC38 mild steel in the absence and in the presence of PW11, PW11Fe and PW11Cu

Very close inhibition percentages were obtained in all three cases $(61.6 \%, 61.1 \%$ and $54.9 \%$ for PW11Fe, PW11 and PW11Cu respectively). As shown in Eq. 9, as soon as the substituted POM oxidises the metal surface, its reduced lacunary counterpart is produced and the observed inhibition mechanism is therefore comparable to that of a lacunary POM.

Despite this interesting performance, spectacular inhibition percentages were not obtained. This was explained by the fact that the action of POMs (lacunary or saturated) as iron oxidants and as consolidating agents of the protective $\mathrm{Fe}$ (II) rich layer remains an unstable equilibrium that controls the extent of the protection. Indeed, for important amounts of POM in the medium, the oxidising action could dominate, resulting in the decrease of the inhibition percentage. This hypothesis was verified when a larger amount of POM (200 ppm) was added to the corrosive solution and led to a less important efficiency compared to the $100 \mathrm{ppm}$ concentration of the equivalent compound (Figure S2). 


\section{Conclusion}

Two lacunary POMs of the Keggin type $\left(\mathrm{K}_{7}\left[\mathrm{PW}_{11} \mathrm{O}_{39}\right] .14 \mathrm{H}_{2} \mathrm{O}\right.$ and $\left.\mathrm{K}_{9}\left[\mathrm{AIW}_{11} \mathrm{O}_{39}\right] .15 \mathrm{H}_{2} \mathrm{O}\right)$ were used as corrosion inhibitors of XC38 steel in $0.1 \mathrm{M} \mathrm{NaCl}$ medium with the aim of reinforcing the oxide layer formed. Electrochemical studies (chronopotentiometry, potentiodynamic studies and electrochemical impedance spectroscopy) have confirmed the anodic inhibitor's character of POMs. The inhibition efficiency obtained with lacunary POMs is greater than that obtained in the presence of their saturated counterparts. Despite this result, the performance of lacunary POMs was not particularly spectacular. The action of POMs as reinforcing agents of the protective Fe(II) rich layer is therefore not enough to provide a highly effective protection against corrosion, but this approach is nonetheless a considerable advance in the understanding of the corrosion inhibition of mild steels by lacunary POMs in $\mathrm{NaCl}$ media.

\section{Experimental Section}

\section{Materials and solutions}

Corrosion experiments were performed on a cylindrical XC38 steel sample purchased from AcierDetailDecoupe and having the following chemical composition (wt \%): $0.36 \mathrm{C}, 0.40 \mathrm{Si}, 0.68 \mathrm{Mn}, 0.045 \mathrm{~S}, 0.40 \mathrm{Cr}, 0.40 \mathrm{Ni}, 0.10 \mathrm{Mo}, 0.045 \mathrm{P}$ and Fe (balance). This steel is very often used in general mechanics because of its good machinability and its mechanical characteristics. For corrosion rate measurements, the lateral surface of the XC38 steel rod was coated with a polytetrafluoroethylene (PTFE) ribbon, leaving a free circular basal working surface of $78.5 \mathrm{~mm}^{2}$. To obtain reproducible measurements, this basal surface (working electrode) was polished on silicon carbide abrasive papers of different grades $(100,1200,2500$, and 4000$)$ from the most abrasive to the least abrasive. A corrosive solution $(0.1 \mathrm{M} \mathrm{NaCl})$ was prepared with commercial $\mathrm{NaCl}$ and deionised water, obtained from a Milli-Q尺 Intregral 5 purification set (Merck KGaA, Darmstadt, Germany). Samples of $\mathrm{K}_{7}\left[\mathrm{PW}_{11} \mathrm{O}_{39}\right] .14 \mathrm{H}_{2} \mathrm{O}$, $\mathrm{K}_{9}\left[\mathrm{AIW}{ }_{11} \mathrm{O}_{39}\right] .15 \mathrm{H}_{2} \mathrm{O}, \mathrm{H}_{3}\left[\mathrm{PW}_{12} \mathrm{O}_{40}\right] .14 \mathrm{H}_{2} \mathrm{O}$ and $\mathrm{H}_{5}\left[\mathrm{AlW}_{12} \mathrm{O}_{40}\right] .15 \mathrm{H}_{2} \mathrm{O}$ were synthesised according to reported synthesis protocols. ${ }^{[24,40-43]}$ All other reagents were of high-purity grade and were used as purchased without further purification. The $\mathrm{pH}$ of the blank solution was 5.63 and those of solutions containing 100 ppm of PW11, 100 ppm of AlW1, 100 ppm of PW12 and 100 ppm of AIW12 was respectively 5.67, 5.68, 3.67 and 5.87.

\section{Electrochemical measurements}

The electrochemical experiments were performed in a thermostated electrochemical cell, with three electrodes. The working electrode was the prepared XC38 steel rod for corrosion studies and glassy carbon for electrochemical characterizations of POMs, a platinum wire as the auxiliary electrode and a saturated calomel electrode (SCE) as the reference electrode. These electrodes were connected to a PARSTAT 4000A AMETEK Potentiostat/Galvanostat/FRA of Princeton Applied Research controlled by the VERSASTUDIO software for electrochemical impedance measurements, for the open circuit and potentiodynamic experiments. Before recording the polarization and EIS curves, the working electrode was first immersed in $\mathrm{NaCl} 0.1 \mathrm{M}$ for 2 hours without inhibitor or in the presence of controlled amounts of inhibitor. The polarization curves were recorded in the potential range -750 to $-100 \mathrm{mV} / \mathrm{SCE}$ at a scan rate of $0.5 \mathrm{mV} / \mathrm{s}$. The corrosion current (Icorr) was obtained by superimposing a straight line along the linear portion of the cathodic or the anodic curve and extrapolating it to the corrosion potential (Ecorr). The polarization resistance $(R p)$ was calculated by taking the inverse of the slope of the current potential curve at $\pm 30 \mathrm{mV}$ relative to the corrosion potential. The inhibition percentages related to the corrosion current measurement and to the polarization resistance measurement were obtained using the following formulas:

$\eta_{\text {Icorr }}=\frac{\text { Icorr' }-I c o r r}{I c o r r^{\prime}}$ and $\eta_{R p}=\frac{R p-R p^{\prime}}{R p}$

where Icorr' and Rp' are the corrosion current and the polarisation resistance without inhibitor, respectively; Icorr and Rp are the corrosion current and the polarisation resistance in the presence of inhibitor, respectively.

EIS curves were recorded at a fixed open circuit potential with $10 \mathrm{mV}$ amplitude, in the frequency range $10^{4} \mathrm{~Hz}$ to $10^{-2} \mathrm{~Hz}$. The EIS curves were fitted using ZsimpWin and EC-Lab softwares to extract useful experimental parameters. The quality of fitting to an equivalent circuit was evaluated by the chi-square value. ${ }^{[44]}$ The inhibition percentages related to the charge transfer resistance were obtained using the following formulas:

$\eta_{R c t}=\frac{R c t-R c t^{\prime}}{R c t}$

where Rct' is the charge transfer resistance without inhibitor and Rct is the charge transfer resistance in the presence of inhibitor.

Field Emission Scanning Electron Microscopy (FESEM) and Energy Dispersive X-Ray Analysis (EDX)

FESEM and EDX analysis was used to investigate the surface morphology and elemental composition of the XC38 steel surface after immersion in $0.1 \mathrm{M} \mathrm{NaCl}$ solutions in the absence and in the presence of POMs at the optimum concentration for 5 hours. FESEM and EDX analysis were performed with a SH-3000 HYROX microscope equipped with an EDX-BRUKER.

\section{Acknowledgements}

The authors acknowledge the support of:

- $\quad$ Agence Universitaire de la Francophonie through the grant AUF-DRACGL-2017-006.

- $\quad$ ISP through the grant o ered to the African Network of Electroanalytical Chemists (ANEC).

- $\quad$ Centre National de la Recherche Scientifique (CNRS). 

B. Larkin, I. Rozenfeld, Prot. Met. 1981, 17, 330-334. D.E. Katsoulis, Chem. Rev. 1998, 98, 359-388.

B. Rani, B.B.J. Basu, Int. J. Corros. 2012, 2012, 1-88

G. Sruthi, K. Shakeela, R. Shanmugam, G. Ranga Rao, Phys. Chem. Chem. Phys. 2020, 22, 3329-3344.

[13] J.J. Borrás-Almenar, E.Coronado, A.Müller, M. Pope, Polyoxometalate molecular science, Springer Science \& Business Media, Netherland, 2003, pp. 3-33.

[14] H.S. Shah, S.A. Joshi, A. Haider, U. Kortz, N.Rehman, J. lqbal, RSC Adv. 2015, 5, 93234-93242.

[15] Y. Kim, S. Shanmugam, ACS Appl. Mater. Interfaces, 2013, 5, 12197-12204.

[16] J. Yin, L. Qi, H. Wang, ACS Appl. Mater. Interfaces, 2011, 3, 4315-4322.

[17] S. Lomakina, T. Shatova, L. Kazansky, Corros. Sci. 1994, 36, 1645-1651.

[18] J. Bonastre, P. Garcés, F. Huerta, C. Quijada, L.G. Andión, F. Cases, Corros. Sci. 2006, 48, 1122-1136.

[19] X. Hu, C. Liang, X. Wu, Mater. Corros. 2011, 62, 444-448.

[20] C. Liang, X. Hu, L. Ma, Mater. Corros. 2007, 58, 39-43.

[21] A. Dolbecq, E. Dumas, C.R. Mayer, P. Mialane, Chem. Rev. 2010, 110, 6009-6048.

[22] G.C. Lica, K.P. Browne, Y. Tong, J. Cluster Sci. 2006, 17, 349-359.

[23] B. Ngouné, M. Pengou, A.M. Nouteza, C.P. Nanseu-Njiki, E. Ngameni, ACS Omega, 2019, 4, 9081-9091.

[24] A. Jürgensen, J.B. Moffat, Catal. Lett. 1995, 34, 237-244.

[25] Y.S. Sangeetha, S. Meenakshi, C.S. Sundaram, Carbohydr. Polym. 2016, 136, 38-45.

[26] L.L. Liao, S. Mo, J.L. Lei, H.Q. Luo, N.B. Li, J. Colloid Interface Sci. 2016, 474, 68-77.

[27] L. Valek, S. Martinez, D. Mikulić, I. Brnardić, Corros. Sci. 2008, 50, 2705-2709.

[28] S. Larfaillou, D. Guy-Bouyssou, F. Le Cras, S. Franger, J. Power Sources, 2016, 319, 139-146.

[29] M.D. Deffo Ayagou, T.T. Mai Tran, B. Tribollet, J. Kittel, E. Sutter, N. Ferrando, C. Mendibide, C. Duret-Thual, Electrochim. Acta, 2018, 282, 775-783

[30] M.N. Kakaei, J. Neshati, Corros. Eng., Sci. Technol. 2019, 54, 413-421.

[31] K. K. Anupama, K. M. Shainy, A. Joseph, J. Bio and Tribo Corros. 2016, 2, 2.

[32] A.L. Chong, J.I. Mardel, D.R. MacFarlane, M. Forsyth, A.E. Somers, ACS Sustainable Chem. Eng. 2016, 4, $1746-1755$.

[33] M. Stratmann, J. Müller, Corros. Sci. 1994, 36, 327-359.

[34] R.M. Cornell, U. Schwertmann, The iron oxides: structure, properties, reactions, occurrences and use, VCH Publisher, Weinheim, 1996, pp. 345-364.

[35] H.B. Gunay, P. Ghods, O.B. Isgor, G.J.C. Carpenter, X. Wu, Appl. Surf. Sci. 2013, 274, $195-202$.

[36] J. Lee, J. Kim, W. Choi, J. Environ. Sci. Technol. 2007, 41, 3335-3340.

[37] B. Keita, L. Nadjo, J. Mol. Catal. A: Chem. 2007, 262,190-215.

[38] B. Keita, I.M. Mbomekalle, L. Nadjo, T.M. Anderson, C.L. Hill, Inorg. Chem., 2004, 43, 3257-3263.

[39] H.K. Ping, F.J. Li, J. Electron Spectrosc. Relat. Phenom. 1997, 83, 93-98.

[40] J.A. Dias, S.C. Loureiro Dias, E. Caliman, J. Bartis, L. Francesconi, Inorg. Synth. 2014, 36, 210-217.

[41] R. Contant, Can. J. Chem. 1987, 65, 568-573..

[42] S. Himeno, M. Takamoto, T. Ueda, J. Electroanal. Chem. 1999, 465, 129-135..

[43] S. Himeno, M. Takamoto, T. Ueda, Bull. Chem. Soc. Jpn. 2005, 78, 1463-1468.

[44] G. Liu, Y. Zhang, M. Wu, Y. Zhang, B. Pang, Int. J. Electrochem. Sci. 2018, 13, 6248-6258. 